\title{
Utilizadores Frequentes da Urgência Pediátrica: Conhecer, Intervir e Analisar - Um Estudo Piloto
}

\author{
Frequent Users of the Pediatric Emergency Department: \\ To Know, To Intervene and To Evaluate - A Pilot Study
}

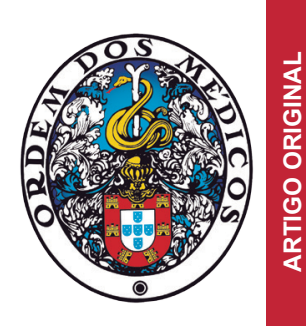

\author{
Marta MARTINS $\triangle^{1}$, Rita MARQUES ${ }^{1}$, Marina SOUSA ${ }^{1}$, Ana VALÉRIO² , Isadora CABRAL ${ }^{2}$, Filomena ALMEIDA ${ }^{3}$ \\ Acta Med Port 2020 May;33(5):311-317 - https://doi.org/10.20344/amp.12769
}

\begin{abstract}
RESUMO
Introdução: Os utilizadores frequentes contribuem para um volume excessivo de admissões no serviço de urgência, prejudicando a qualidade dos serviços prestados. O objetivo deste estudo foi identificar e caracterizar os utilizadores frequentes da urgência pediátrica, traçar um plano de intervenção e avaliar a sua eficácia.

Material e Métodos: Estudo de intervenção, incluindo crianças e adolescentes menores de 16 anos, com mais de 10 admissões num ano na urgência pediátrica de um hospital de nível II. Foi criado um modelo de intervenção com uma equipa multidisciplinar e um gestor de caso, responsável pela execução do plano de intervenção individualizado. Após um ano de intervenção foi avaliada a evolução do número de admissões.

Resultados: Identificámos 90 utentes com o perfil de utilizador frequente, com 1182 admissões. Apenas 17,3\% das admissões eram referenciadas. A maioria das admissões foi considerada não urgente/pouco urgente (59\%). Foram incluídas na intervenção 82 crianças/adolescentes. Após um ano de intervenção verificou-se uma diminuição significativa do número de admissões, com uma redução média de $62 \%$ em 78 participantes.

Discussão: A maioria das admissões dos utilizadores frequentes foi considerada não urgente/pouco urgente, evidenciando a ausência de indicação clínica para observação hospitalar. Os resultados da intervenção parecem ser favoráveis, com uma redução do número de admissões.

Conclusão: Esta população de utilizadores frequentes da urgência pediátrica constitui um grupo heterogéneo, com problemas de complexidade variável. Um modelo de intervenção multidisciplinar e individualizado, com um gestor de caso pode contribuir para reduzir o recurso excessivo à urgência e melhorar a prestação de cuidados de saúde a estas crianças.

Palavras-chave: Mau Uso de Serviços de Saúde; Pediatria; Portugal; Serviço de Urgência Hospitalar
\end{abstract}

\section{ABSTRACT}

Introduction: Frequent users contribute to an excessive volume of admissions in the emergency department, impairing the quality of healthcare services. The aim of this study was to identify and characterize the population of frequent users of the pediatric emergency department, establish an individual plan of intervention and evaluate its efficacy.

Material and Methods: Intervention study including children and adolescents younger than 16 years, with more than 10 visits in a year to an emergency department of a secondary care hospital. An intervention program was created with a multidisciplinary team and a case manager, who was responsible for the execution of the individual plan of intervention. One year later, the number of visits after the intervention was assessed.

Results: We identified 90 patients with a frequent user profile, with 1182 visits. Only $17.3 \%$ of the visits had a previous referral. Most of the visits were considered non urgent/less urgent (59\%). The intervention included 82 children/adolescents. One year later, there was a significant reduction in the number of visits: median reduction of $62 \%$ in 78 participants.

Discussion: Most of the visits by frequent users were considered non urgent/less urgent, reflecting a clinically unjustified use of the emergency department. The intervention seems to be effective as the number of visits decreased.

Conclusion: This population of frequent users seems to be a heterogeneous group with different problems and levels of complexity. A multidisciplinary and individual intervention, with a case manager, might contribute to reduce the excessive use of the emergency department and improve the provision of health care services to these children.

Keywords: Emergency Service, Hospital; Health Services Misuse; Pediatrics; Portugal

\section{INTRODUÇÃO}

$\mathrm{O}$ recurso à urgência tem aumentado de forma considerável nos últimos anos em vários países por todo o Mundo. ${ }^{1-3}$ Ainda assim, Portugal é o país da Organização para a Cooperação e Desenvolvimento Económico (OCDE) com maior número de admissões per capita no serviço de urgência (SU), com mais de 70 admissões por cada 100 habitantes, e os utentes em idade pediátrica constituem um dos grupos com maior taxa de utilização. ${ }^{3}$

Além da sobrelotação da urgência, da sobrecarga dos profissionais e dos gastos inerentes, esta utilização excessiva tem impacto na qualidade dos serviços prestados à população. Em adultos, alguns estudos têm mostrado que doentes observados repetidamente na urgência apresentam pior prognóstico e maior taxa de mortalidade. ${ }^{4}$ De forma similar, em idade pediátrica tem surgido evidência de associação a um pior controlo de doença crónica e seguimento mais irregular a longo prazo. ${ }^{5-7}$

Dois fatores distintos contribuem de forma significativa

1. Serviço de Pediatria. Hospital Garcia de Orta. Almada. Portugal.

2. Unidade de Saúde Familiar S. João do Pragal. Agrupamentos de Centro de Saúde Almada-Seixal. Almada. Portugal.

3. Serviço Social. Hospital Garcia de Orta. Almada. Portugal.

$\square$ Autor correspondente: Marta Martins. marta.martins@campus.ul.pt

Recebido: 01 de setembro de 2019 - Aceite: 16 de outubro de 2019 | Copyright @ Ordem dos Médicos 2020 
para este elevado volume de doentes na urgência pediátrica (UP): as admissões injustificadas e os utilizadores frequentes (UF). ${ }^{4,8-10}$

Neste sentido tem sido dada uma atenção cada vez maior a este conjunto de utentes denominados de 'utilizadores frequentes, ${ }^{11-13}$ e definidos como utentes com utilização repetida e recorrente à urgência ao longo de um determinado período.

A vigilância e monitorização destes doentes constitui um potencial indicador da qualidade dos serviços de saúde, quer a nível hospitalar, quer a nível dos cuidados de saúde primários (CSP). ${ }^{14,15} \mathrm{~A}$ identificação e caracterização dos utilizadores frequentes reveste-se assim de enorme importância no sentido de criar estratégias de intervenção apropriadas e eficazes.

Vários tipos de intervenção têm sido elaborados junto destes grupos de utentes ${ }^{16,17}$ globalmente divididas em três tipos diferentes: gestão de caso, plano individual de intervenção e partilha de informação. ${ }^{16}$ Os dois primeiros tipos consistem numa abordagem multidisciplinar de intervenção através da criação de um plano individual de cuidados para cada utente. No modelo de gestão de caso, é atribuído um contacto único para cada utente - o gestor de caso, responsável pelo seu acompanhamento. O terceiro tipo de intervenção implica a criação de estratégias de comunicação entre os prestadores de cuidados de saúde de cada utente a nível hospitalar e dos cuidados de saúde primários, com o objetivo de coordenar a abordagem ao doente e torná-la mais consistente e uniforme.

Contudo estes diversos modelos de intervenção foram aplicados essencialmente em adultos, não existindo até à data estudos exclusivamente com doentes pediátricos.

Assim, o objetivo deste trabalho foi identificar e caracterizar os utilizadores frequentes da nossa UP, traçar um plano individual de intervenção e avaliar a sua eficácia.

\section{MATERIAL E MÉTODOS}

Foi desenvolvido um estudo de intervenção no qual foram admitidas todas as crianças e adolescentes consideradas utilizadores frequentes da UP de um hospital de nível II. Foram excluídos adolescentes com idade igual ou superior a 16 anos uma vez que o seguimento ao longo do período de estudo seria dificultado pela transição para os cuidados de adultos a partir dos 18 anos. Foi considerada a idade à data de recrutamento dos participantes, isto é, a 1 de janeiro de 2018.

De acordo com uma das definições propostas na literatura para a idade pediátrica e que é também a mais consensual na população adulta, foi definido UF o utente com mais de 10 vindas à UP no período de um ano (janeiro a dezembro de 2017).

Os dados foram obtidos a partir da revisão dos processos clínicos, tendo sido recolhida informação relativamente a caracterização sociodemográfica, história médica pregressa e seguimento habitual (cuidados de saúde primários e/ou consulta hospitalar) e caracterização das admissões: número de admissões, número de admissões referencia- das e não referenciadas, nível de prioridade atribuída na triagem, realização de meios complementares de diagnóstico e/ou terapêutica endovenosa e número de internamentos. A triagem realizada na UP atribui a cada utente a prioridade segundo o protocolo da triagem de Manchester em quatro níveis: não urgente, pouco urgente, urgente ou muito urgente.

Foi criada uma equipa multidisciplinar composta por profissionais de saúde hospitalares e dos cuidados de saúde primários (médicos, enfermeiros, assistente social), responsável pela apreciação individual de cada utente e estabelecimento de um plano de intervenção personalizado e adaptado. A intervenção seguiu o modelo de gestão de caso, no qual foi definido um gestor de caso para cada utente e que constituiu o principal responsável pela execução do plano individual desse utente. O plano de intervenção, de carácter multidisciplinar, envolveu primordialmente a aproximação aos cuidados de saúde primários, referenciação/acompanhamento em consulta hospitalar e/ou avaliação e acompanhamento social.

O primeiro passo da intervenção foi a identificação dos utentes e a sua respetiva caracterização no que diz respeito à história clínica e principais problemas médicos e sociais.

Desta primeira análise sobressaíram dois grupos relativamente distintos: um grupo com seguimento exclusivamente em cuidados de saúde primários composto maioritariamente por crianças saudáveis e um grupo de utentes com seguimento hospitalar, com maior proporção de doença crónica.

No primeiro grupo, a aproximação dos utentes aos CSP e o ensino e a educação para saúde dos pais e cuidadores, sobretudo no que diz respeito à abordagem da doença aguda e das principais patologias em idade pediátrica constituíram os pilares essenciais da intervenção. Relativamente ao segundo grupo, a intervenção passou também pela abordagem na consulta hospitalar, procurando identificar as principais dificuldades na abordagem da doença crónica e reforçar o ensino aos pais quanto ao tratamento da respetiva patologia e as indicações para observação médica na UP.

Nalguns casos foram identificados fatores de risco social como desemprego, baixa escolaridade dos pais, imigração/clandestinidade e patologia mental dos pais. Estes utentes foram submetidos a uma avaliação social e familiar e respetiva intervenção nessa área.

A intervenção teve início em abril de 2018 e um ano após o início da sua implementação foi avaliado o resultado da mesma através da evolução do número de admissões ao longo desse período. Foi realizada uma monitorização das admissões na UP não só no nosso hospital como noutros hospitais a nível nacional através da consulta da plataforma de dados de saúde (PDS) e as mesmas foram contabilizadas na análise estatística. Em apenas um doente não foi possível obter esses dados.

Todos os dados recolhidos foram tratados de forma confidencial e foi requerido consentimento informado a 
todos os participantes. O estudo foi aprovado pela Comissão de Ética do Hospital Garcia de Orta (HGO).

\section{Análise estatística}

Os dados foram submetidos a análise descritiva para cálculo de médias, medianas, desvio-padrão (DP) e proporções e os resultados são apresentados maioritariamente em percentagem. A amostra foi avaliada quanto à normalidade da sua distribuição com recurso ao teste de Shapiro-Wilk. As diferenças de proporções entre grupos foram testadas com recurso ao teste de Fisher e ao teste do qui-quadrado.

Para análise dos resultados da intervenção cada doente admitido constituiu o seu próprio controlo histórico, tendo em conta os dados prévios à intervenção. O número de admissões na UP pré e pós intervenção foi comparado através do teste de Wilcoxon. Considerou-se como estatisticamente significativo um valor de $p<0,05$. A análise estatística foi realizada através de software informático: Microsoft Excel ${ }^{\circledR}$ e GraphPad Prism $5^{\circledR}$.

\section{RESULTADOS}

\section{Caracterização da amostra}

Foram identificados 90 utentes com mais de 10 admis- sões na UP no ano de 2017, com um total de 1182 admissões, o que corresponde a $2,4 \%$ do total de admissões no mesmo período. Destes foram excluídos oito utentes pela idade ( $\geq 16$ anos), restando 82 participantes, com um total de 1063 admissões. À data do início do estudo a mediana de idades dos utentes incluídos foi de dois anos, com $84 \%$ dos doentes (69/82) com uma idade igual ou inferior a três anos (Tabela 1). Setenta e três por cento dos utentes (60/82) eram do sexo masculino. A idade média dos pais foi de 35 anos (pai) e 33 anos (mãe); a respetiva escolaridade está representada na Tabela 1 .

Quanto à história médica pregressa, 48\% (39/82) dos UF tinham uma doença crónica (Tabela 1) e destes a maioria apresentava patologia respiratória (30/39) sobretudo sibilância recorrente/asma (29/30). Em cerca de metade $(48 \%, 19 / 39)$ dos doentes com patologia crónica a maioria das admissões estavam relacionadas com a sua patologia de base; entre o grupo da sibilância recorrente/asma essa proporção subiu para $62 \%$ (18/29).

Em termos de seguimento, 55\% (45/82) mantinham apenas seguimento ao nível dos cuidados de saúde primários e 45\% (37/82) tinham acompanhamento em consulta hospitalar. A maioria dos utentes $(64,6 \%, 52 / 82)$ pertencia a uma unidade de saúde familiar (USF) enquanto os

Tabela 1 - Características sociodemográficas e presença de doença crónica dos utilizadores frequentes consoante o tipo de seguimento (Grupo 1: seguimento exclusivo nos cuidados de saúde primários e Grupo 2: seguimento hospitalar) e totalidade da amostra.

\begin{tabular}{|c|c|c|c|}
\hline Características & $\begin{array}{r}\text { Grupo } 1 \\
(n=45) \\
\end{array}$ & $\begin{array}{r}\text { Grupo } 2 \\
(\mathrm{n}=37) \\
\end{array}$ & $\begin{array}{c}\text { Total } \\
(\mathrm{n}=\mathbf{8 2})\end{array}$ \\
\hline \multicolumn{4}{|l|}{ Idade (anos) } \\
\hline $1-3$ & $40(88,9)$ & $29(78,4)$ & $69(84,1)$ \\
\hline $4-6$ & $4(8,9)$ & $4(10,8)$ & $8(9,8)$ \\
\hline $7-9$ & $1(2,2)$ & $2(5,4)$ & $3(3,7)$ \\
\hline $10-12$ & $0(0)$ & $1(2,7)$ & $1(1,2)$ \\
\hline $13-15$ & $0(0)$ & $1(2,7)$ & $1(1,2)$ \\
\hline \multicolumn{4}{|l|}{ Género } \\
\hline Feminino & $13(28,9)$ & $9(24,3)$ & $22(26,8)$ \\
\hline Masculino & $32(71,1)$ & $28(75,7)$ & $60(73,2)$ \\
\hline \multicolumn{4}{|l|}{ Escolaridade Pai } \\
\hline Sem escolaridade & $0(0)$ & $0(0)$ & $0(0)$ \\
\hline $1^{\circ} \mathrm{Ciclo}$ & $5(11,1)$ & $2(5,4)$ & $7(8,5)$ \\
\hline $2 / 3^{\circ}$ Ciclo & $15(33,3)$ & $15(40,5)$ & $30(36,6)$ \\
\hline Ensino Secundário & $8(17,8)$ & $11(29,7)$ & $19(23,2)$ \\
\hline Ensino Superior & $10(22,2)$ & $2(5,4)$ & $12(14,6)$ \\
\hline Desconhecido & $7(15,6)$ & $7(18,9)$ & $14(17,1)$ \\
\hline \multicolumn{4}{|l|}{ Escolaridade Mãe } \\
\hline Sem escolaridade & $0(0)$ & $1(2,7)$ & $1(1,2)$ \\
\hline $1^{\circ}$ Ciclo & $0(0)$ & $0(0)$ & $0(0)$ \\
\hline $2 / 3^{\circ}$ Ciclo & $20(44,4)$ & $16(43,2)$ & $36(43,9)$ \\
\hline Ensino Secundário & $5(11,1)$ & $12(32,4)$ & $17(20,7)$ \\
\hline Ensino Superior & $13(28,9)$ & $4(10,8)$ & $17(20,7)$ \\
\hline Desconhecido & $7(15,6)$ & $4(10,8)$ & $11(13,4)$ \\
\hline Doença crónica & $9(20 \%)$ & $30(81,1 \%)$ & $39(47,6)$ \\
\hline Patologia respiratória & 9 & 21 & 30 \\
\hline Patologia neurológica & 0 & 2 & 2 \\
\hline Patologia nefro-urológica & 0 & 2 & 2 \\
\hline Patologia pedopsiquiátrica/desenvolvimento & 0 & 2 & 2 \\
\hline Patologia gastrointestinal & 0 & 1 & 1 \\
\hline Multipatologia & 0 & 2 & 2 \\
\hline
\end{tabular}


Tabela 2 - Proporção de doença crónica, número de internamentos e admissões: comparação entre grupos consoante tipo de seguimento (Grupo 1: seguimento exclusivo nos cuidados de saúde primários e Grupo 2: seguimento hospitalar)

\begin{tabular}{lrrr}
\hline & $\begin{array}{r}\text { Grupo 1 } \\
(\mathbf{n}=\mathbf{4 5})\end{array}$ & $\begin{array}{c}\text { Grupo 2 } \\
(\mathbf{n}=\mathbf{3 7})\end{array}$ & Teste Fisher \\
\hline Doença crónica & $9(20 \%)$ & $30(81,1 \%)$ & $p<0,001$ \\
Internamento & $12(2,1 \%)$ & $24(4,8 \%)$ & $p=0,0174$ \\
Admissões pré-intervenção & 564 & 499 & \\
$\quad$ Urgentes & $186(33,0)$ & $250(50,1)$ & $p<0,001$ \\
$\quad$ Não urgentes & $378(67,0)$ & $249(49,9)$ & \\
\hline
\end{tabular}

estantes (35,4\%, 29/82) eram acompanhados em unidades de cuidados de saúde personalizados (UCSP). Foram identificados sete utentes que, apesar de residirem na área de influência do hospital, encontravam-se inscritos em unidades de cuidados de saúde primários não pertencentes ao Agrupamento de Centros de Saúde (ACES) Almada-Seixal. Dos 82 utentes, 12 não tinham médico de família atribuído $(14,6 \%)$.

Cada utente teve uma mediana de quatro consultas nos CSP ao longo do ano de 2017 (mínimo: 0; máximo: 19). Nove utentes $(11 \%)$ também tinham um padrão de utilização muito frequente nos CSP, com 10 ou mais consultas nesse período.

\section{Caracterização das admissões hospitalares}

Os utilizadores frequentes apresentaram uma mediana de 17 admissões por utente por ano, 12 no ano de 2017 ( mínimo $=11$, máximo $=29)$. Do total de admissões em 2017 apenas 17,3\% (184/1063) tinham uma referenciação prévia pelo médico assistente ou pela linha Saúde 24. A maioria das admissões foi considerada não urgente ou pouco urgente (admissões não urgentes/ pouco urgentes: $627 / 1063,59 \%$; admissões urgentes/ muito urgentes: 436/1063, 41\%). Em 15,1\% das admissões (160/1063) foram realizados exames complementares de diagnóstico e em $1,7 \%$ terapêutica endovenosa (18/1063). Verificaram-se 36 internamentos, o que corresponde a uma taxa de internamento de $3,4 \%$.

Cerca de metade dos utentes (44/82, 53,7\%) apresentaram vindas à UP por um motivo recorrente (mínimo: 3; máximo: 12$)$, sendo que a maioria destes $(28 / 44,63,6 \%)$

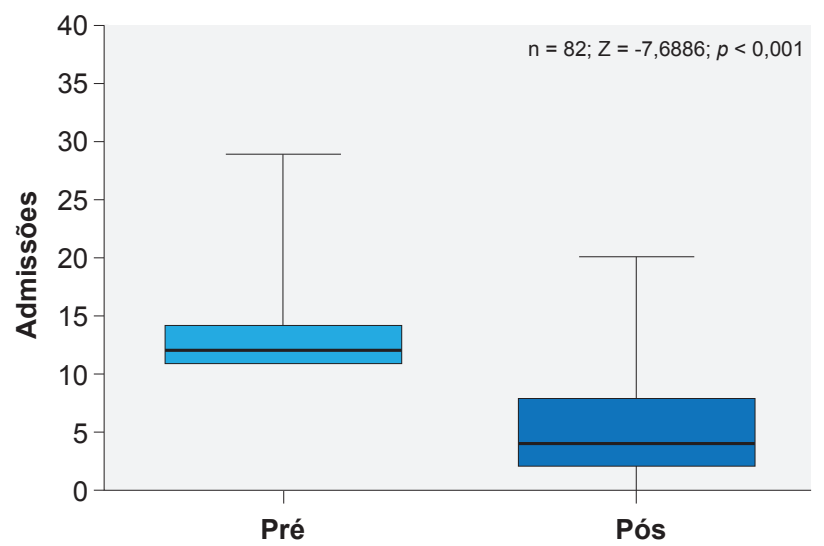

Figura 1 - Comparação do número de admissões na urgência pediátrica dos utilizadores frequentes pré e pós intervenção tinham já um seguimento em consulta hospitalar por esse motivo.

O grupo dos utentes com seguimento hospitalar apresentaram maior proporção de doença crónica (81\% vs $20 \%$, $p<0,0001)$, maior número de admissões urgentes $(50 \%$ vs $33 \%, p<0,0001)$ e maior taxa de internamento $(4,8 \%$ vs $2,1 \%, p=0,0174)$ - Tabela 2. Apesar disso, para o total das admissões da amostra (1063) cada grupo contribuiu de igual forma: 499 admissões pelo grupo com seguimento hospitalar (47\%) e 564 admissões pelo grupo sem seguimento hospitalar (53\%).

\section{Evolução após intervenção}

Ao longo do ano em que foi realizada a intervenção verificou-se uma diminuição no número de admissões em $95 \%$ dos utentes (78/82), com uma redução, em média, de $62 \%$ do número de vindas (DP: 25,4). Em cerca de dois terços (56/82; 68\%) essa redução foi superior a 50\%. A mediana de admissões foi de quatro (mínimo: 0; máximo: 20), diferença estatisticamente significativa em relação ao ano prévio à intervenção (mediana de 12), $z=-7,6886, p<0,0001$ (Fig. 1).

Em apenas quatro casos se verificou uma evolução no sentido contrário. Assim, do total de utentes admitidos no estudo, $10(12 \%)$ persistiram como UF.

A metade dos utentes (6/12) que não tinham médico assistente atribuído foi-lhes designado um médico de família. Três dos sete utentes que estavam inscritos em USF/ UCSP não pertencentes aos ACES Almada/Seixal efetuaram a transferência.

Relativamente à evolução do número de consultas nos

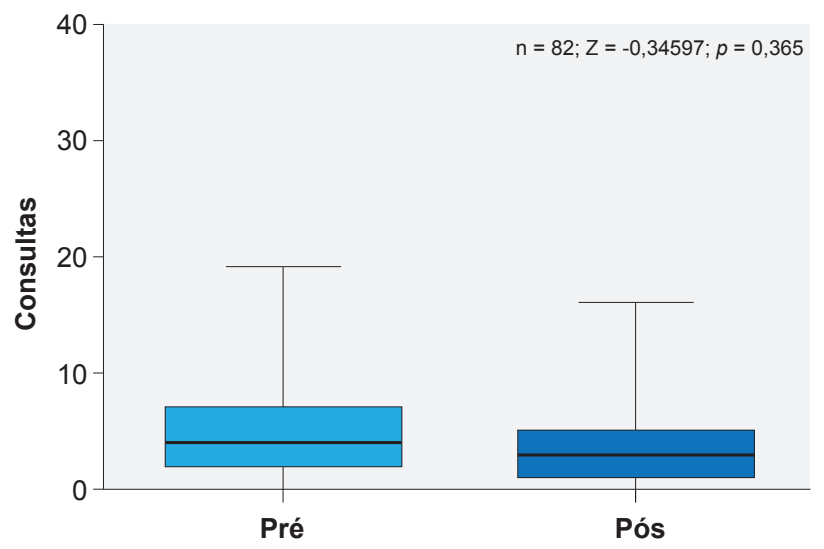

Figura 2 - Comparação do número de consultas nos cuidados de saúde primários dos utilizadores frequentes pré e pós intervenção 
CSP (Fig. 2), em 60\% ( $n=49)$ dos utentes verificou-se uma diminuição do número de consultas, com uma redução média de $60 \%$. Nos restantes $40 \%(n=33)$ houve um aumento ou estabilização desse número. Quando comparados estes dois grupos verificamos que o grupo em que houve tendência para maior número de vindas aos CSP era constituído por utentes com uma mediana de consultas menor no ano prévio à intervenção (mediana: uma consulta/ano versus seis consultas/ano; $p<0,0001)-$ Fig. 3. Dos nove utentes que eram considerados também UF nos CSP, em oito registou-se uma diminuição significativa do número de consultas (mediana de 12 consultas pré-intervenção versus seis consultas pós intervenção; $z=-2,55, p=0,005$ ).

\section{DISCUSSÃO}

\section{Conhecer os utilizadores frequentes}

À semelhança do que tem sido descrito em estudos semelhantes, verificámos uma proporção importante de utilizadores frequentes da UP. ${ }^{8-10}$ A maioria das admissões foi considerada não urgente/ pouco urgente e não exigiu nenhum tipo de exames complementares de diagnóstico ou de terapêutica i.v., evidenciando a ausência de indicação clínica para a recorrência a uma urgência hospitalar. A taxa de internamento neste grupo de utentes foi sobreponível à taxa global de internamento. Neuman et al num estudo multicêntrico demonstraram de forma semelhante que cerca de um terço de todas as admissões na UP não implicavam terapêutica, exames complementares de diagnóstico ou internamento e que essa proporção aumentava à medida que a frequência de utilização recorrente da UP aumentava. $^{8}$

A idade parece ser um fator importante e que condiciona um maior número de admissões. García et al mostraram que $88 \%$ dos utilizadores frequentes tinham uma idade igual ou inferior a três anos, ${ }^{18}$ resultado sobreponível ao nosso. Seguin et al demonstraram haver uma associação entre a idade mais jovem e a utilização recorrente da

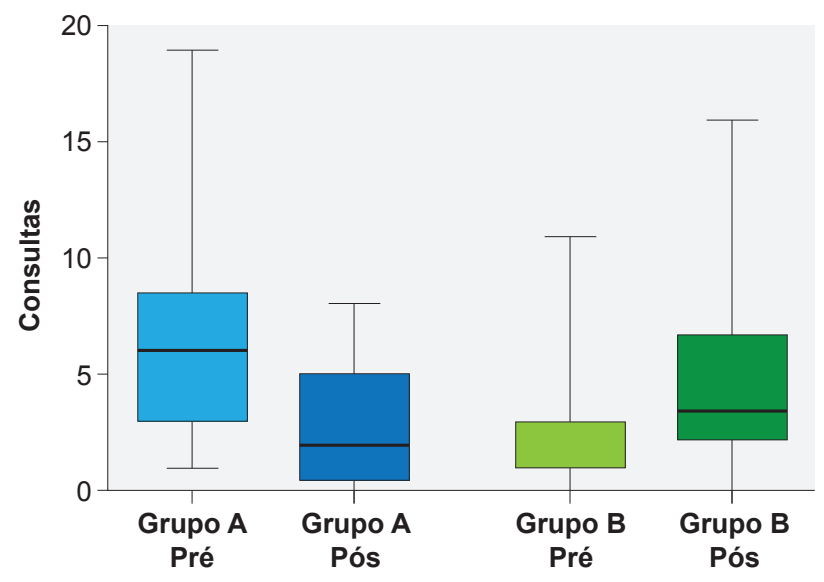

Figura 3 - Comparação do número de consultas nos cuidados de saúde primários dos utilizadores frequentes pré e pós intervenção por grupo: grupo $A$ - utentes com redução do número de consultas pós-intervenção e grupo $\mathrm{B}$ - utentes com aumento do número de consultas pós-intervenção
UP, com um maior número de admissões até aos três anos e uma redução importante desse número posteriormente, a partir da idade escolar. ${ }^{19} \mathrm{~A}$ maior frequência de doenças infeciosas nesta idade a par de alguma inexperiência e ansiedade por parte dos pais podem explicar estes resultados.

A existência de uma patologia crónica não parece ser um fator tão preponderante como se esperaria já que menos de metade dos UF tinham uma doença crónica e destes apenas cerca de metade tinham uma proporção importante de admissões relacionadas com a sua patologia de base. Ainda assim, o grupo dos doentes com asma e sibilância recorrente constituiu um grupo expressivo e com um número importante de admissões por agudização. Alpern et al constataram igualmente que apenas $40 \%$ das admissões dos utilizadores frequentes estavam relacionadas com uma patologia crónica e que a asma era o diagnóstico mais frequente neste grupo de doentes. ${ }^{9}$ Outros estudos têm demonstrado que efetivamente os doentes com asma tem uma elevada taxa de recorrência à Urgência em contexto de agudizações e vários fatores têm sido apontados: baixa adesão terapêutica, informação insuficiente acerca da abordagem da crise e aplicação ineficaz do plano de tratamento na agudização. ${ }^{20,21}$

O acesso limitado aos cuidados de saúde primários tem sido um fator comumente associado a um maior número de admissões na UP. ${ }^{22,23}$ Contudo, a maioria destes utentes dispõe de um médico de família atribuído e dois terços pertencem a uma unidade de saúde familiar, fatores favoráveis a um melhor acesso aos cuidados de saúde primários. Efetivamente $11 \%$ dos utentes apresentavam um perfil de UF também nos CSP previamente à intervenção, sugerindo que, pelo menos neste grupo, o acesso aos CSP não é um fator determinante.

Assim, esta população de utilizadores frequentes parece constituir um grupo heterogéneo, com problemas diversos e de complexidade variável, o que demonstra a necessidade de uma abordagem individualizada e adaptada.

\section{Intervir e avaliar}

Os resultados ao longo da intervenção parecem ser favoráveis, com uma redução significativa do número de admissões na UP da maioria dos utentes. De igual forma a evolução da utilização dos CSP parece ter sido globalmente mais adequada: nos utentes que tinham um número de consultas reduzido previamente à intervenção houve um aumento do recurso aos CSP e nos utentes que tinham um recurso excessivo houve a tendência inversa.

Alguns fatores como o avançar da idade e a consequente menor frequência de doenças infeciosas agudas podem ter contribuído também para a redução das admissões e dificultam a interpretação dos resultados.

Ainda assim, parece-nos que este modelo de intervenção com um gestor de caso se revelou eficaz, indo de encontro ao descrito noutros estudos em adultos. ${ }^{24-28}$ Apesar da população de UF adultos ter características francamente diferentes da nossa (grande proporção de doença crónica, 
patologia mental, toxicodependência e problemas socioeconómicos), a maioria dos estudos com intervenções do tipo gestão de caso revelou uma diminuição significativa do número de admissões no serviço de urgência. ${ }^{29}$

A ausência de estudos de intervenção em idade pediátrica não nos permite comparar os nossos resultados com outras experiências e modelos de intervenção. No entanto, consideramos que este modelo pela proximidade com cada utente e a adequação da intervenção às suas características individuais permite uma abordagem multidisciplinar eficaz com vista à mudança de padrão de utilização dos cuidados de saúde e, acima de tudo, a uma prestação de cuidados mais adequada e abrangente.

\section{Limitações}

Vários outros fatores têm sido apontados como potencialmente favorecedores desta utilização frequente da UP como a proximidade da urgência hospitalar, conveniência do horário de funcionamento permanente, observação 'imediata' e perceção dos pais/cuidadores de maior disponibilidade de recursos diagnósticos e terapêuticos a nível hospitalar. Nenhum destes fatores foi devidamente avaliado neste estudo embora possam ter um peso importante neste padrão de utilização frequente da UP.

Outra das limitações deste estudo é inerente a um estudo de coorte histórico em que os próprios sujeitos são utilizados como controlos. Quando são selecionados sujeitos com valores extremos num dado parâmetro (como o número elevado de admissões na UP), existe a possibilidade desse valor diminuir naturalmente sem intervenção graças a outros fatores. Apenas um estudo aleatorizado e controlado ultrapassaria essa limitação.

Até à data o período de seguimento foi de apenas um ano, o que pode ser insuficiente para averiguar a redução sustentada do recurso à urgência. A continuidade deste projeto e uma nova avaliação posteriormente permitirão avaliar a eficácia a longo prazo e quais os fatores determinantes para a mesma.

\section{CONCLUSÃO}

Neste estudo encontrámos um número significativo de utilizadores frequentes, com uma proporção importante de admissões na UP. A maioria destas admissões corresponderam a situações pouco ou nada urgentes, com uma baixa taxa de internamento e, portanto, de utilização injustificada dos cuidados hospitalares.

Esta intervenção do tipo gestão de caso parece ser eficaz na abordagem a estes utentes e respetivos pais e cuidadores, embora estes resultados devam ser futuramente validados em estudos aleatorizados e controlados. Não existem até à data outros estudos de intervenção em populações pediátricas com este perfil de utilizador frequente que nos permitam fazer uma comparação dos nossos resultados.

A afluência excessiva e inapropriada à urgência consome recursos, prejudica a capacidade de resposta dos serviços a situações urgentes e influencia negativamente as atitudes dos profissionais de saúde. Assim, pelo impacto que tem na qualidade dos serviços prestados este é um problema que carece de melhor caracterização a nível nacional. A implementação de medidas e estratégias de intervenção a nível nacional e local é urgente. A nível local a criação de um modelo de intervenção, articulando os cuidados de saúde primários com os cuidados hospitalares, pode constituir uma forma de resolução do problema com vista à redução do número de admissões na UP e respetivos custos e, acima de tudo à melhoria da prestação de cuidados de saúde a estas crianças.

\section{PROTEÇÃO DE PESSOAS E ANIMAIS}

Os autores declaram que os procedimentos seguidos estavam de acordo com os regulamentos estabelecidos pelos responsáveis da Comissão de Investigação Clínica e Ética e de acordo com a Declaração de Helsínquia da Associação Médica Mundial.

\section{CONFIDENCIALIDADE DOS DADOS}

Os autores declaram ter seguido os protocolos do seu centro de trabalho acerca da publicação de dados.

\section{CONFLITOS DE INTERESSE}

Os autores declaram não ter conflitos de interesses relacionados com o presente trabalho.

\section{FONTES DE FINANCIAMENTO}

Este trabalho não recebeu qualquer tipo de suporte financeiro de nenhuma entidade no domínio público ou privado.

\section{REFERÊNCIAS}

1. Niska R, Bhuiya F, Xu J. National hospital ambulatory medical care survey: 2007 emergency department summary. Natl Health Stat Report. 2010:1-31.

2. Doan Q, Genuis E, Yu A. Trends in use in a Canadian pediatric emergency department. CJEM. 2014;16:405-10.

3. Berchet C. Emergency care services. OECD Health Working Papers. Paris: Organization for Economic Cooperation and Development; 2015.

4. Hoot N, Aronsky D. Systematic review of emergency department crowding: causes, effects, and solutions. Ann Emerg Med. 2008;52:12636.e1.

5. Hostetler M, Mace S, Brown K, Finkler J, Hernandez D, Krug S, et al. Emergency department overcrowding and children. Pediatr Emerg Care. 2007;23:507-15.

6. Timm N, Ho M, Luria J. Pediatric emergency department overcrowding and impact on patient flow outcomes. Acad Emerg Med. 2008;15:832-7.

7. Christakis D, Mell L, Koepsell T, Zimmerman F, Connell F. Association of lower continuity of care with greater risk of emergency department use and hospitalization in children. Pediatrics. 2001;107:524-9.

8. Neuman M, Alpern E, Hall M. Characteristics of recurrent utilization in pediatric emergency departments. Pediatrics. 2014;134:e1025-31.

9. Alpern E, Clark A, Alessandrini E, Gorelick M, Kittick M, Stanley R, et al. Recurrent and high-frequency use of the emergency department by pediatric patients. Acad Emerg Med. 2014;21:365-73.

10. Cabey W, MacNeill E, White L, James Norton H, Mitchell A. Frequent pediatric emergency department use in infancy and early childhood. Pediatr Emerg Care. 2014;30:710-7. 
11. Ruger J, Richter C, Spitznagel E, Lewis L. Analysis of costs, length of stay, and utilization of emergency department services by frequent users: implications for health policy. Acad Emerg Med. 2004;11:1311-7.

12. Pines J, Asplin B, Kaji A, Lowe R, Magid D, Raven M, et al. Frequent users of emergency department services: gaps in knowledge and a proposed research agenda. Acad Emerg Med. 2011;18:e64-9.

13. LaCalle E, Rabin E. Frequent users of emergency departments: the myths, the data, and the policy implications. Ann Emerg Med. 2010;56:42-8.

14. Friedlaender E. Patterns of health care use that may identify young children who are at risk for maltreatment. Pediatrics. 2005;116:1303-8.

15. Guenther E, Knight S, Olson L, Dean J, Keenan H. Prediction of child abuse risk from emergency department use. J Pediatr. 2009;154:272-7.

16. Soril L, Leggett L, Lorenzetti D, Noseworthy T, Clement F. Reducing frequent visits to the emergency department: a systematic review of interventions. PLoS One. 2015;10:e0123660.

17. Moe J, Kirkland S, Rawe E, Ospina M, Vandermeer B, Campbell S, et al. Effectiveness of interventions to decrease emergency department visits by adult frequent users: a systematic review. Acad Emerg Med. 2017;24:40-52.

18. Rivas García A, Manrique Martín G, Butragueño Laiseca L, Mesa García S, Campos Segura A, Fernández Iglesia V et al. Frequent users in paediatric emergency departments. Who are they? Why do they consult? An Pediatr. 2017;86:67-75.

19. Seguin J, Osmanlliu E, Zhang $X$, Clavel V, Eisman $H$, Rodrigues $R$ et al. Frequent users of the pediatric emergency department. CJEM. 2017;20:401-8

20. Sullivan P, Vahram G, Abhishek K, Prakash N, Friedman H, Benjamin O. Trends in asthma control, treatment, healthcare utilization and expenditures among children in the United States by place of residence:
2003-2014. J Allergy Clin Immunol Pract. 2019;7:1835-42.

21. To T, Zhu J, Ryckman K, Gershon A. Risk factors for return to the emergency department for asthma: a population-based study. J Allergy Clin Immunol Pract. 2018;6:1907-13.

22. Fieldston E, Alpern E, Nadel F, Shea J, Alessandrini E. A qualitative assessment of reasons for nonurgent visits to the emergency department. Pediatr Emerg Care. 2012;28:220-5.

23. Zickafoose J, DeCamp L, Prosser L. Association between enhanced access services in pediatric primary care and utilization of emergency departments: a national parent survey. J Pediatr. 2013;163:1389-95.

24. Okin R, Boccellari A, Azocar F, Shumway M, O'Brien K, Gelb A, et al The effects of clinical case management on hospital service use among ED frequent users. Am J Emerg Med. 2000;18:603-8.

25. Pope D, Fernandes CM, Bouthillette F, Etherington J. Frequent users of the emergency department: a program to improve care and reduce visits. CMAJ. 2000;162:1017-20.

26. Shumway M, Boccellari A, O'Brien K, Okin R. Cost-effectiveness of clinical case management for ED frequent users: results of a randomized trial. Am J Emerg Med. 2008;26:155-64.

27. Crane S, Collins L, Hall J, Rochester D, Patch S. Reducing utilization by uninsured frequent users of the emergency department: combining case management and drop-in group medical appointments. J Am Board Fam Med. 2012;25:184-91.

28. Chiang C, Lee C, Tsai T, Li C, Lee W, Wu K. Dynamic internetmediated team-based case management of high-frequency emergency department users. J Emerg Crit Care Med. 2014;21:161-6.

29. Soril L, Leggett L, Lorenzetti D, Noseworthy T, Clement F. Reducing frequent visits to the emergency department: a systematic review of interventions. PLoS One. 2015;104:e0123660. 